\title{
Efficient Sub Area Tree Routing Protocol (Estr) For Collision Avoidance in Mobile Adhoc Networks
}

\author{
G.Vetrichelvi ${ }^{1}$, Dr.G.Mohankumar ${ }^{2}$ \\ ${ }^{I}$ Department of Electronics and Communication Engineering, PARK college of Engineering and Technology, \\ Coimbatore. \\ ${ }^{2}$ Department of Mechanical Engineering, PARK college of Engineering and Technology, Coimbatore.
}

\begin{abstract}
Efficient Sub area tree routing, is named after its performance, where the whole network is divided into many subareas, such that they form sub area trees. The root nodes are identified by manual configuration or auto-discovery process. Initially a root node establishes sub area trees and each node either forms a sub area tree or become a interconnect node. ESTR is a hierarchical routing protocol and does not maintain the routing information in every node consistently.It exhibits both the behaviour of proactive and on-demand routing protocols. The simulation results shows that the throughput value of the protocol is considerably found to be maximum compared to AODV protocol which leads to the avoidance of collision, reduces the delay, energy consumption and the data transmission time.
\end{abstract}

Keywords: ESTR, hierarchical routing protocol, throughput, collision avoidance.

\section{Introduction}

Mobile Adhoc networks do not depend on a static infrastructure and the structure varies due to the mobility of the nodes. These networks play an important role in the fields which does not utilize or use the fixed infrastructure such as data acquisition, conferences, military communications etc. Each node of the network acts as a router which identifies and maintains routes to othernodes which is not in the direct transmission range, by forminga multiple hop path and the packets are forwarded by store and forward methodology.

Many research works is going on in this area and number of protocols have been proposed based on the major challenges of this routing in mobile adhoc networks such as frequently changing network topology, power transmitted, minimum bandwidth, delay and energy consumption. Some of the work done on routing protocols has been given below[1].

In proactive protocols [2-7], routes between every two nodes are established in advance even if no data transmission is on demand. This is implemented by a node periodically updating its routing information and every node eventually has consistent and up-to-date global routing information for the entire network. This approach has the advantages of timely exchanging network information such as available bandwidth, delay, topology etc. and supporting real-time services. But it is not suitable for largescale networks since many unused routes still need to be maintained and the periodic updating may incur overwhelming processing and communication overhead. The on-demand protocol (e.g. [8-11]) is more efficient because each node tries to reduce routing overhead by only sending routing packets when needed for data transmission and a route is released when the data transmission is finished. However, when link breakage is detected due to failure or node mobility, which often occurs in multi-hop wireless ad hoc networks, the delay and overhead of route reconstruction may be significant. As to flat routing and hierarchical routing, this is a classification according to the network structure underlying routing protocols. In flat routing protocol, there is a peer-to-peer relationship between every two nodes and each node participating in routing plays an equal role. Routing algorithm is easily realized. However, it is inferior scalability and limits network's scale in a certain extent. For example, AODV [8], DSR [9] and DSDV [2] are typical flat routing protocols. The hierarchical routing protocol divides the whole network into many logical areas and different routing strategies are used inside and outside the logical area. Compared with flat routing protocol, the hierarchical routing protocol possesses better scalability and is propitious to support large-scalenetworks.

This paper presents a new hierarchical routing protocol which provides, low delay, low overhead, energy consumption and collision avoidance. The main concept of the ESTR is to construct sub area trees depending upon the environment and then the whole network will be divided into many sub areas. ESTR possesses the advantages of both ondemand routing protocol and proactive routing protocol, which provides the delay and low overhead of the reactive and proactive methods. The description of the protocol operation and performance are briefly described in the following chapters. Finally the protocol is compared with another protocol and the results and conclusions are provided. 


\section{Efficient Subarea Tree Routing}

The main operation of this routing protocol is based on the establishment of subarea tree by having a selected root node as origin which results in the formation of logical subarea. The entire network consists of many sub area trees finally.

The stages involved in the ESTR protocol is as follows

i) Identification and Interconnection of root nodes.

ii) Creation of subarea trees.

iii)Maintenance of subarea trees.

iv)Routing process.

\section{i) Identification and Interconnection of root nodes.}

Initially a node with a unique address or an ID and its type is configured as initial node. Each node has a unique ID. The central node of a future subarea is the root node and it can be identified by auto-discovery process or static method. Static method is the manual method to configure the root nodes and to decide the related or necessary parameters for them.

The auto-discovery process is a complex process which involves many steps. The first step is to provide a regulation or the condition which the roots must satisfy such as ID number, energy, transaction capability, number of neighbour nodes and other values. Next a node is selected randomly from the network as the source node and it sends the Auto Root Discovery Request(ARDR) message which includes the regulation. The nodes which satisfy the regulation will be considered as the root nodes and sends their own details such as the type and ID.All the root nodes will record the root nodes' information.

After identification, interconnection of the root nodes is done by building the routing information between every two root nodes. This can be done by using the routing protocols such as AODV,DSR,FSR,ZRP etc....The first node which the route passes through becomes the interconnect node. Both root node and interconnect node will maintain the routing table with the information.

\section{ii) Creation of Subarea trees.}

The subarea trees are created using the tree nodes that join the subarea trees. The tree node possess the information of its node ID,node type,depth, father node, number of child node and routing list. The depth is the hop count between the tree node and its route node.The root nodes and tree nodes periodically transmits Subarea Tree Creation Message(STCM). The initial node which receives the STCM will consider itself as tree node and sets the father node as the nodeID from the STCM. The depth of the tree node is set as the depth of the STCM plus one. Next, it returns a Data Updating Message(DUM) which involves the destination node ID, source node ID, no.of offspring nodes and node routing list to the father node. Then the father node updates the child node list, no.of.offspring nodes and the routing list. This process continues for each and every father node up to root nodes.

\begin{tabular}{|l|l|l|l|l|}
\hline $\begin{array}{l}\text { Type of } \\
\text { Message }\end{array}$ & Node ID & Type of Node & Number of offspring & Depth \\
\hline
\end{tabular}

Fig.1. Format of STCM

\begin{tabular}{|l|l|l|l|l|}
\hline $\begin{array}{l}\text { Type of } \\
\text { Message }\end{array}$ & $\begin{array}{l}\text { Number of } \\
\text { offspring }\end{array}$ & Routing list & Destination ID & Source ID \\
\hline
\end{tabular}

Fig.2. Format of DUM

If more than one STCM is received by an initial node it will select the STCM according to the regulation and discard the others.If an initial node cannot join a subarea tree after a particular time $\mathrm{T}$, if forms a connection with the interconnected nodes with one node as root node.It also notifies other root nodes that it has become a root node. Finally all initial nodes will join in subarea trees to become tree nodes. The STCM received by root node will be discarded.

\section{iii) Subarea Tree Maintenance}

The subarea tree is maintained by the information between the child node and the father node. The child node will identify the existence of the father node by the STCM sent periodically. Father node identifies the existence of child node by exchanging information between them. If there is no information from the child node the father node will send a "Hello Message" to the child node, if there is no reply for a particular time period it will delete the child node from child node list and update the number of offspring node and routing list, then inform its father node by DUM. 
If the relationship between the child node and father node is failed the child node will configure it as "initial node", inform all its child nodes to configure their node type as initial node and will send as Father Node Releasing Message (FNRM). All the initial nodes will start to join in subarea trees.

\begin{tabular}{|ll|l|l|l|}
\hline $\begin{array}{l}\text { Type of } \\
\text { Message }\end{array}$ & $\begin{array}{l}\text { Tree nodes } \\
\text { released }\end{array}$ & Destination ID & Source ID \\
\hline
\end{tabular}

Fig.3. Format of FNRM

\section{iv)Routing process}

In the routing process root node knows the routing information of all the tree nodes in the hierarchical network formed after the establishment of subarea trees. The first tier consists of root nodes and interconnects nodes and the second tier is each sub area tree. The two tiers have different routing parameters.Proactive routing is used in inter subarea. The routing process is as follows. First if the data packet's destination node is of its same offspring node, the node will forward the packet to the destination directly.If it is not its own offspring, the node will forward the packet to the father node.If root node also has its routing information Route search Message(RSM) is send to other root nodes. It includes the message type, destination root node ID, source root node ID and data packet's destination Id and acknowledgement. Other root nodes which knows the routing information will return a Route Detection Message(RDM) to the source root node which will forward the data packet to the interconnect node which knows the route to the destination node.

\begin{tabular}{|l|l|l|l|l|}
\hline $\begin{array}{l}\text { Type of } \\
\text { Message }\end{array}$ & $\begin{array}{l}\text { Source root } \\
\text { ID }\end{array}$ & Dest root ID & Data destination ID & Ack \\
\hline
\end{tabular}

Fig.4. Format of RDM/RSM

\section{v)Example}

A simplified example is illustrated[1] in figure 5 and there are 22 network nodes. At first all the nodes are "equal" and eachnode is "initial node" with a unique ID. The line between twonodes denotes a wireless link and these two nodes cancommunicate directly.

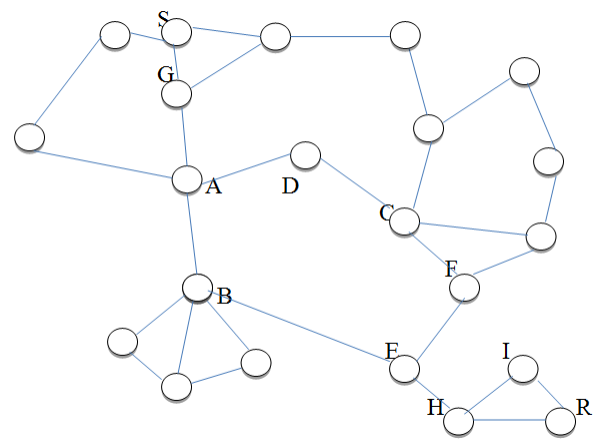

Fig. 5.Original network

Assume the condition of becoming a root node in thisexample is that the number of neighbors is more than or equal4. Let node $\mathrm{S}$ broadcasts $A R D R$ message and nodes $\mathrm{A}, \mathrm{B}, \mathrm{Cwill}$ become root nodes. These three root nodes will broadcasttheir information so that they can know each other. Afterwardsthrough existing routing protocol such as AODV, theywill build routing information (routing metrics: hop count orburthen is least in this example): $\mathrm{A} \leftrightarrow \mathrm{B} ; \mathrm{A} \leftrightarrow \mathrm{D} \leftrightarrow \mathrm{C} ; \mathrm{B} \leftrightarrow \mathrm{E} \leftrightarrow \mathrm{F} \leftrightarrow \mathrm{C}$. During this procedure, nodes $\mathrm{D}, \mathrm{E}, \mathrm{F}$ willbecome "interconnect node". Root nodes A, B, C start the establishing process ofsubarea trees by broadcasting STCM. The initial nodesreceiving this message will join in subarea trees and become"tree node" simultaneously. Then tree nodes also send outSTCM periodically. All the initial nodes except nodes H, I, Rwill join in subarea trees successfully through such a process.Under this circumstances, interconnect node E will beupgraded to become root node and it will gain other rootnodes' information from the nearest root node B as well asnotify other root nodes of its own information. And then nodesE, H, I, R form a new subarea tree. At last the entire networkis composed of four subarea trees illustrated as figure 6 andtwo tiers of network structure is obvious. 


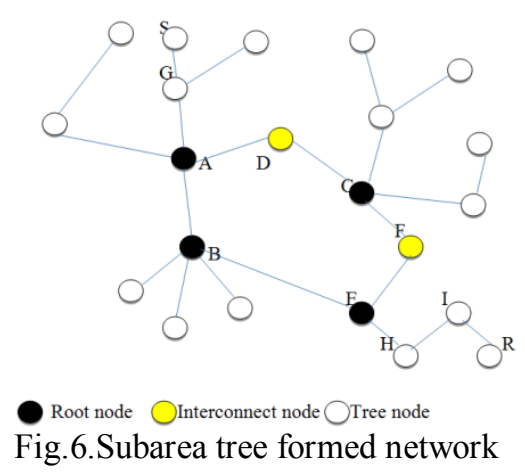

Assume node $\mathrm{S}$ wants to send data packets to node R.Firstly $\mathrm{S}$ sends the data packets to its father node G. Node Gfinds node $\mathrm{R}$ is not its offspring node and sends the datapackets to root node A. Root node A will lookup routinginformation for node $\mathrm{R}$, but it cannot find the route. And then A sends $R S M$ to root nodes $\mathrm{B}, \mathrm{C}, \mathrm{E}$ and node $\mathrm{E}$ will answer thismessage with $R D M$ because it knows node $\mathrm{R}$ is its offspringnode. Node $\mathrm{A}$ will send data packets to node $\mathrm{E}$ while itreceives $R D M$. Finally node $\mathrm{R}$ can receive the data packetsthrough nodes $\mathrm{E}$ and $\mathrm{H}$.

\section{i) Simulation software}

\section{Performance Analysis}

We used NS2.3 (Network Simulation 2.3) to evaluate theperformance of STR. The simulation modeled a network ina $2500 \mathrm{~m} \times 2500 \mathrm{~m}$ area with 30 mobile nodes. Radiotransmission range is 250 meters. The mobility of eachnode is arranged from $2 \mathrm{~m} / \mathrm{s}$ to $8 \mathrm{~m} / \mathrm{s}$, and the pause time ofthe mobile nodes is zero. Traffic sources are continuous bitrate (CBR) with the rate of $15 \mathrm{kbit} / \mathrm{s}$. The source-destinationpairs are randomly selected over the network.

\section{ii)Simulation Result}

The simulation result is obtained by comparing the performance of STR with AODV.The green line shows the plot for STR routing protocol whereas the red line denotes the AODV routing protocol. The graph shown above is plotted by comparing both the STR and AODV routing protocol by considering the parameter: throughput. Since the throughput efficiency for STR routing protocol is maximum as compared to the AODV routing protocol the chance of collision, delay, energy consumption, time consumption, data sending time will be more in the AODV routing protocol. Thus the proposed system with STR routing protocol is more efficient than the system with AODV routing protocol.

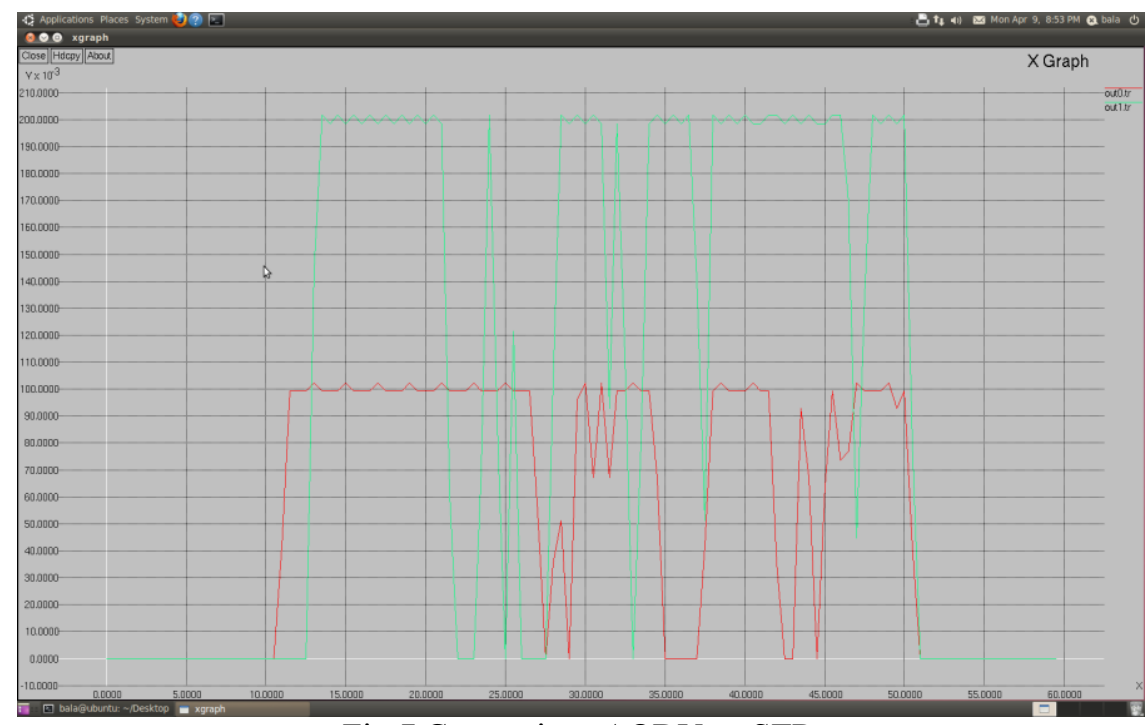

Fig.7.Comparison AODV vs STR

\section{Conclusion}

We have proposed Subarea Tree Routing (STR), a novel routing protocol for collision avoidance in mobile ad-hoc networks. One of the main reason behind implementing STR protocol is that bit does not incur heavy overhead as it maintains the routing information in MANET. In STR, many nodes are formed into 
different group each consisting a header such as the load along the nodes will be less compared to other routing protocols. Thus lesser the load in the nodes, the chance to occur collision in mobile ad-hoc networks minimized. Thus by achieving collision avoidance in MANET using STR protocol, it helps to enhance the efficiency and speed. At the same time, it reduced intrusions in the network. Our ns-2 based simulation has confirmed the advantages of STR and demonstrated a significant routing efficiency and scalability improvement.

\section{Journal Papers}

\section{References}

[1] Liu Gui-kai, Shan Chun-li, Wei Gang, Wang Hong-jiang,Subarea Tree Routing in Multi-hop Wireless Ad hoc Networks,IEEE ,pp 1695-1699,2008.

[2] Johnson D, Hu Y and Maltz D. "The dynamic source routing protocol (DSR) for mobile ad hoc networks for IPv4”. IETF Internet RFC4728, February 2007.

[3] Ogier R, Templin F and Lewis M. "Topology Broadcast based on Reverse-Path Forwarding (TBRPF)". IETF Internet RFC3684, February 2004.

[4] Clausen T and Jacquet P. “Optimized Link State Routing Protocol (OLSR)”. IETF Internet RFC3626, October 2003.

[5] C.E. Perkins, E.M. Belding-Royer, and I. Chakeres, "Ad Hoc OnDemand Distance Vector (AODV) Routing," IETF Internet RFC3561, July 2003.

[6] Park V and Corson S. "Temporally-Ordered Routing Algorithm (TORA) Version 1 Functional Specification", IETF Internet draft (draft-ietfmanet- tora-spec-04.txt), July 2001.

[7] C.-K. Toh, "Associativity-Based Routing For Ad Hoc Mobile Networks," WLPers. Commun. J., Special Issue on Mobile Networking and Computing Systems,Kluwer, vol. 4, no. 2, Mar. 1997, pp. 103-139. [11] Haas Z J, and Peariman M R. "The performance of query control schemes for the zone routing protocol". IEEHACM Transactions on Networking, Vol. 9, No. 4, Aug. 2001 , pp. $427-438$.

\section{Workshops}

[1] Pei Guangyu, Gerla Mario, Hong X. "LANMAR: Landmark Routing for Large Scale Wireless Ad hoc Networks with Group Mobility”, Proceedings of IEEE/ACM Workshop on Mobile Ad hoc networking \& Computing, MobiHOC'00, Boston, MA, USA, 2000,MA,USA: 2000, pp. 11-18.

[2] G. Pei, M. Gerla and T.W. Chen, "Fisheye State Routing in Mobile Ad Hoc Networks," In Proceedings of the 2000 ICDCS Workshops, Taipei, Taiwan, Apr. 2000, pp. D71-D78.

\section{Proceedings papers}

[1] Pei G, Gerla M and Hong X Y et al. “A Wireless Hierarchical Routing Protocol with Group Mobility”. Proc. IEEE WCNC '99, New Orleans, LA, Sept. 1999, pp.1538-1542 1699

[2] J.J. Garcia-Luna-Aceves and M. Spohn, "Source-Tree Routing in Wireless Networks," Proc. IEEE Int'l Conf. Network Protocols (ICNP), 1999, pp.273-282.

[3] Chiang C C and Gerla M. "Routing and Multicast in Multihop, Mobile Wireless Networks". Proc. IEEE ICUPC '97, San Diego, CA, Oct. 1997, pp.546-551.

[4] C.E. Perkins, "Highly Dynamic Destination-Sequenced Distance-Vector Routing (DSDV) for Mobile Computers," Proc. ACM SIGCOMM, pp. 234-244, 1994. 